\title{
A Common Framework for Blind Multistage Multiuser Receivers of DS-CDMA in Frequency-Selective Fading Channels
}

\author{
Sau-Hsuan Wu, Urbashi Mitra and C.-C. Jay Kuo \\ Department of Electrical Engineering \\ University of Southern California \\ Los Angeles, California 90089-2564 USA \\ E-mail: \{sauhsuaw, ubli\}@usc.edu, cckuo@sipi.usc.edu
}

\begin{abstract}
-
The multistage (MS) Wiener filter proposed in [1] is extended to blind maximum likelihood (ML) multiuser detection for direct-sequence (DS) code-division multiple access (CDMA) systems. This multistage ML detector is shown to share the same structure with the MS minimum-mean squared error (MMSE), minimum output energy (MOE) and best linear unbiased estimator (BLUE) filter banks (FBs) derived in $[2,3]$, each with a distinctive output scaling matrix. Based on this result, a common framework is proposed for the implementation and analysis of coherent maximum ratio combining (MRC) and non-coherent differential equal gain combining (EGC) of these receivers. A generic bit error rate (BER) formula is provided for receivers constructed within this common framework. The BER floors for EGC receivers are analyzed and compared to those of the MRC schemes presented in [4]. Based on simulation results, the heterogeneous EGC BLUE-ML receiver exhibits the best performance due to the fact that BLUE-FB is essentially the ML channel estimator and ML-FB is the ML symbol detector for the linear Gaussian system models of DS-CDMA systems.
\end{abstract}

\section{INTRODUCTION}

Reduced-rank linear filtering based on the multistage Wiener filter (MSWF) of [1] has proven to be an effective data detection scheme for multiuser direct-sequence (DS) code-division multiple-access (CDMA) systems [5]. A key feature of the reduced rank MSWF implementation is that near full-rank performance is achieved with relatively few stages. The bulk of the analysis of such schemes for DSCDMA has focused on additive white Gaussian noise channels [5] (with a large system analysis in [6]). We have proposed a class of linear multistage (MS) estimator/detector structures for the case of multipath Rayleigh fading using the following cost functions: minimum mean-squared error (MMSE), minimum mean output energy (MOE) and the best linear unbiased estimation criterion (BLUE) [2,3]. Herein, the multistage implementation is further extended to blind maximum likelihood (ML) data detection.

In the absence of real-time channel estimation for fast fading channels, non-coherent differential equal gain combining (EGC) can be employed [7]. In such schemes, there is a segment of the detector that acts as an implicit channel estimator and another segment which acts as the data detector. We deem receiver structures which use the same method for implicit channel estimation and detection as homogeneous schemes. We have designed homogeneous EGC algorithms for MS MMSE/MOE/BLUE cost functions in $[2,3]$. In the current work, we evaluate heterogeneous combinations such as MS BLUE-MMSE and MS BLUE-ML based receivers, motivated by the fact that the BLUE estimator is the minimum variance unbiased (MUV) estimator for the Gaussian linear system model.

Exploiting the similarity in implementation structure for

This research has been funded in part by the Integrated Media Systems Center, a National Science Foundation Engineering Research Center, Cooperative Agreement No. EEC-9529152, and in part by the National Science Foundation under Grant NSF/ANI-0087761. the various MS receivers, we develop a common framework for the implementation of both EGC and maximal ratio combining (MRC) schemes. A further contribution of the work herein is the derivation of a generic bit error rate (BER) formula for receivers constructed with this common framework. Limiting performance, by way of BER error floors, are analyzed and the resultant behavior is compared and contrasted for EGC and MRC methods. We can show that the BERs for each class of combining methods are equivalent for the case of flat Rayleigh fading; this coincides with prior results showing similar behavior for the output signal-to-interference plus noise ratio (SINR) [4].

\section{System Model}

A standard model for asynchronous DS-CDMA modulated with binary phase shift keying (BPSK) is considered. Assume the maximum path delay for each user is less than one symbol interval $T_{s}$. After pulse matched filtering and chip rate sampling, the discrete-time received signal vector y obtained by collecting $\mathrm{N}$ consecutive samples, ( $\mathrm{N}$ is the spreading gain), is given by [7]

$$
\begin{aligned}
& \mathbf{y}(m)=\sum_{k=1}^{K}\left[\mathbf{S}_{\mathbf{k}+} \mathbf{A}_{\mathbf{k}} \Gamma_{k}(m) b_{k}(m)\right. \\
& \left.+\mathbf{S}_{\mathbf{k}-} \mathbf{A}_{\mathbf{k}} \Gamma_{k}(m) b_{k}(m-1)\right]+\mathbf{n}(m),
\end{aligned}
$$

where $K$ is the number of users, $\mathbf{A}_{k}=\operatorname{diag}\left(\left[A_{k 1}, \ldots, A_{k L_{k}}\right]\right)$, $\mathbf{S}_{\mathbf{k}+}=\left[\mathbf{s}_{k 1}^{+}, \ldots, \mathbf{s}_{k L_{k}}^{+}\right], \mathbf{S}_{\mathbf{k}-}=\left[\mathbf{s}_{k 1}^{-}, \ldots, \mathbf{s}_{k L_{k}}^{-}\right]$and $\Gamma_{k}=$ $\left[\gamma_{k 1}, \ldots, \gamma_{k L_{k}}\right]^{T}$. The number of paths of user $k$ is $L_{k}$, $A_{k l}$ is the amplitude of the signal on path $l$ of user $k$, $\mathbf{s}_{k l}^{+}$and $\mathbf{s}_{k l}^{-}$are the partial spreading codes corresponding to the current bit $b(m)$ and the previous bit $b(m-1)$ over the sampling interval, respectively. The filtered noise vector $\mathbf{n}(m)$ is complex Gaussian distributed with zero mean and covariance matrix, $N_{0} \mathbf{I}$. Finally, the discretetime fading process $\gamma_{k l}(m)$ over the sampling interval $T_{s}$ is a complex zero-mean Gaussian process that satisfies $E\left\{\gamma_{k_{1} l_{1}}^{*}(m) \gamma_{k_{2} l_{2}}(m)\right\}=\delta\left(k_{1}-k_{2}\right) \delta\left(l_{1}-l_{2}\right)$, with the autocorrelation of two adjacent samples defined as $\rho=E\left[\gamma_{k_{1} l_{1}}^{*}(m) \gamma_{k_{1} l_{1}}(m-1)\right]$. For analysis, the system model is alternatively written as

$$
\begin{aligned}
\mathbf{y}(m) & =\mathbf{S}_{1+} \mathbf{A}_{1} \Gamma_{1}(m) b_{1}(m)+\mathbf{I}_{1}(m) \\
\text { where } \mathbf{I}_{1}(m) & \equiv \sum_{k=2}^{K}\left[\mathbf{S}_{k+} \mathbf{A}_{k} \Gamma_{k}(m) b_{k}(m)\right. \\
& \left.+\sum_{k=1}^{K} \mathbf{S}_{k-} \mathbf{A}_{k} \Gamma_{k}(m) b_{k}(m-1)\right]+\mathbf{n}(m) \cdot(2)
\end{aligned}
$$

The interference vector $\mathbf{I}_{1}$ is the aggregate of the multiple access interference (MAI), inter symbol interference (ISI) and the complex Gaussian noise; with auto-covariance matrix defined as $\mathbf{C}_{1} \equiv E\left(\mathbf{I}_{1} \mathbf{I}_{1}^{H}\right)$. The vector $\mathbf{I}_{1}$ is also complex Gaussian distributed because $\mathbf{S}_{k+}$ and $\mathbf{S}_{k-}$ are deter- 
ministic and $\Gamma_{k}$ is a complex Gaussian vector. For simplicity, we use $L$ to denote $L_{1}$ in the remainder of this paper.

\section{ML AND MMSE Detectors}

To develop the proposed ML blind detector, the aggregation of the MAI, ISI and AWGN channel is treated as colored Gaussian noise. By the system model of (2), the probability density function of $\mathbf{y}$ conditioned on $\Gamma_{1} b_{1}$ is

$$
\begin{aligned}
& \mathcal{P}\left(\mathbf{y} \mid \Gamma_{1} b_{1}\right)=\frac{1}{(2 \pi)^{N} \operatorname{det}\left(\mathbf{C}_{1}\right)} \times \\
& \exp \left\{-\left(\mathbf{y}-\mathbf{S}_{1+} \mathbf{A}_{1} \Gamma_{1} b_{1}\right)^{H} \mathbf{C}_{1}^{-1}\left(\mathbf{y}-\mathbf{S}_{1+} \mathbf{A}_{1} \Gamma_{1} b_{1}\right)\right\}
\end{aligned}
$$

The ML symbol detector is given by

$$
\begin{aligned}
\hat{b}_{1}(m) & =\operatorname{sgn}\left\{\operatorname{Re}\left[\Gamma_{1}^{H}(m) \mathbf{A}_{1} \mathbf{S}_{1+}^{H} \mathbf{C}_{1}^{-1} \mathbf{y}(m)\right]\right\} \\
& \equiv \operatorname{sgn}\left\{\operatorname{Re}\left[\Gamma_{1}^{H}(m) \omega_{m l}^{H} \mathbf{y}(m)\right]\right\} .
\end{aligned}
$$

This blind ML detector is similar in structure to the MMSE multiuser detector given by [8]

$$
\omega_{m m s e}=\arg \min _{\omega} E\left\|b_{1} \Gamma_{1}-\omega^{H} \mathbf{y}\right\|^{2}=\mathbf{R}^{-1} \mathbf{S}_{1+} \mathbf{A}_{1},
$$

where $\mathbf{R}=E\left(\mathbf{y} \mathbf{y}^{H}\right)$.

Conditioned on $\Gamma_{1} b_{1}$, the filter bank which maximizes the instantaneous output SINR of the form [9]

$$
\arg \min _{\omega} \frac{E^{2}\left(\omega^{H} \mathbf{y} \mid \Gamma_{1} b_{1}\right)}{\operatorname{Var}\left(\omega^{H} \mathbf{y} \mid \Gamma_{1} b_{1}\right)}=\arg \min _{\omega} \frac{\omega^{H} \mathbf{S}_{1+} \mathbf{A}_{1} \Gamma_{1} \Gamma_{1}^{H} \mathbf{A} \mathbf{S}_{1+}^{H} \omega}{\omega^{H} \mathbf{C}_{1} \omega}
$$

is actually the ML filter bank $\omega_{m l}=\mathbf{C}_{1}^{-1} \mathbf{S}_{1+} \mathbf{A}_{1} \Gamma_{1}$, with the optimal SINR equal to $\Gamma_{1}^{H} \mathbf{A}_{1} \mathbf{S}_{1+}^{H} \mathbf{C}_{1}^{-1} \mathbf{S}_{1+} \mathbf{A}_{1} \Gamma_{1}$. Taking the expectation with respect to $\Gamma_{1}$ of a Rayleigh fading channel yields the unconditional average SINR:

$$
E\left(\Gamma_{1}^{H} \mathbf{A}_{1} \mathbf{S}_{1+}^{H} \mathbf{C}_{1}^{-1} \mathbf{S}_{1+} \mathbf{A}_{1} \Gamma_{1}\right)=\operatorname{tr}\left(\mathbf{A}_{1} \mathbf{S}_{1+}^{H} \mathbf{C}_{1}^{-1} \mathbf{S}_{1+} \mathbf{A}_{1}\right) .
$$

We can also evaluate the instantaneous output SINRs for the MMSE, MOE and BLUE filter banks by substituting the corresponding $\omega$ into (5). However, obtaining closedform formulae for the unconditional SINRs seems to be intractable. If we assume a MRC filter bank of the form $\omega \Gamma_{1}$, where $\omega$ is not a function of the fading coefficient vector $\Gamma_{1}$, we can evaluate the output SINR, defined as

$$
S I N R \equiv \frac{E\left(\Gamma_{1}^{H} \omega^{H} \mathbf{y} \mid b_{1}=1\right)^{2}}{\operatorname{Var}\left(\Gamma_{1}^{H} \omega^{H} \mathbf{y} \mid b_{1}=1\right)} .
$$

Such a SINR measure is widely used for performance evaluations in fading channels. The filter bank which maximizes this output SINR can be shown equal to $\omega_{m m s e}$ with the optimal output SINR equal to

$$
\operatorname{tr}\left(\mathbf{A}_{1} \mathbf{S}_{1+}^{H} \mathbf{R}^{-1} \mathbf{S}_{1+} \mathbf{A}_{1}\right) .
$$

As will be shown later, the BERs associated with the MMSE and ML filter banks with EGC and MRC combinings are functions of the eigenvalues of the kernel matrices: $K_{m l} \equiv \mathbf{A}_{1} \mathbf{S}_{1+}^{H} \mathbf{C}_{1}^{-1} \mathbf{S}_{1+} \mathbf{A}_{1}$ and $K_{m m s e} \equiv$ $\mathbf{A}_{1} \mathbf{S}_{1+}^{H} \mathbf{R}^{-1} \mathbf{S}_{1+} \mathbf{A}_{1}$. Note that the two SINRs just discussed are evaluated by taking the traces of these kernel matrices. In the sequel, the average of SINR in the sense of (5) will be referred to as the average SINR, and the average of SINR in the sense of (7) will be referred to as the output SINR.

\section{Multistage ML Detector}

Motivated by the multistage implementation for the MMSE-FB in [2], the ML-FB can also factorize into a multistage fashion. Assume the matrix $\mathbf{S}_{1+}$ is of full column

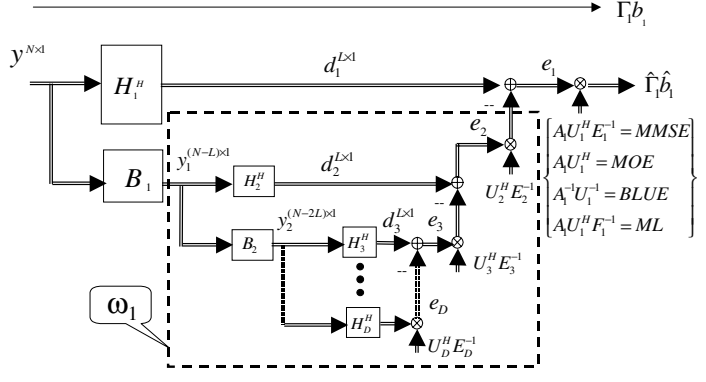

Fig. 1. The common structure for the D-stage multiuser receivers in multipath channels. The output scaling matrix is $\mathbf{A}_{1} \mathbf{U}_{1}^{H} \mathbf{E}_{1}^{-1}$ for MMSE-FB, $\mathbf{A}_{1} \mathbf{U}_{1}^{H}$ for MOE-FB, $\mathbf{A}_{1}^{-1} \mathbf{U}_{1}^{-1}$ for BLUE-FB and $\mathbf{A}_{1} \mathbf{U}_{1}^{H} \mathbf{F}_{1}^{-1}$ for ML-FB. The objective of estimation is $\Gamma_{1} b_{1}$.

rank. Following the development of a transformation matrix, $\mathbf{T}$ in [2], $\mathbf{S}_{1+}$ can be factored by the Gram-Schmidt process into $\mathbf{S}_{1+}=\mathbf{H}_{1} \mathbf{U}_{1}$, where the column space of $\mathbf{H}_{1}$, denoted as $\mathcal{H}_{1}$, is an orthogonal space spanning the column space of $\mathbf{S}_{1+}$ with $\mathbf{H}_{1}^{H} \mathbf{H}_{1}=\mathbf{I}$. Similarly, we can find the orthogonal complement of $\mathcal{H}_{1}$, denoted as $\mathcal{B}_{1}$, which spans the row space of the so-called blocking matrix $\mathbf{B}_{1}$ such that $\mathbf{B}_{1} \mathbf{H}_{1}=\mathbf{0}$. Now, transforming the received signal $\mathbf{y}(m)$ with the unitary matrix $\mathbf{T} \equiv\left[\frac{\mathbf{H}_{1}^{H}}{\mathbf{B}_{1}}\right]$ yields

$\mathbf{T y}=\mathbf{T S}_{\mathbf{1}+} \mathbf{A}_{\mathbf{1}} \Gamma_{1}(m) b_{1}(m)+\mathbf{T I}_{1}(m)+\mathbf{T n}(m)$ Define $\mathbf{C}_{T}=\mathbf{T} \mathbf{C}_{1} \mathbf{T}^{H}$ and $\mathbf{S}_{T}=\mathbf{T} \mathbf{S}_{1+}$. The ML detector operating on the transformed received signal is given by

$$
\omega_{m l}=A_{1} \mathbf{S}_{T}^{H} \mathbf{C}_{T}^{-1} \mathbf{T} \mathbf{y}(m)=A_{1} \mathbf{S}_{1+}^{H} \mathbf{C}_{1}^{-1} \mathbf{y}(m) .
$$

The equality can be easily verified since $\mathbf{T}^{H}=\mathbf{T}^{-1}$. Similar to the cases of the MS-MMSE/MOE/BLUE filter banks, the unitary transformation does not alter the detection result. Employing the procedure in [3], it can be shown that

$$
\mathbf{C}_{T}^{-1}=\left[\begin{array}{c|c}
\mathbf{F}_{1}^{-1} & -\mathbf{F}_{1}^{-1} \mathbf{r}_{\mathbf{y}_{1} \mathbf{d}_{1}}^{\mathbf{R}_{\mathbf{y}_{1}}} \\
\hline-\mathbf{R}_{\mathbf{y}_{1}}^{-1} \mathbf{r}_{\mathbf{y}_{1} \mathbf{d}_{1}} \mathbf{F}_{1}^{-1} & \Delta
\end{array}\right],
$$

where $\mathbf{d}_{1} \equiv \mathbf{H}_{1}^{H} \mathbf{y}$ and $\mathbf{y}_{1} \equiv \mathbf{B}_{1} \mathbf{y}, \mathbf{r}_{\mathbf{y}_{1} \mathbf{d}_{1}} \equiv E\left(\mathbf{y}_{1} \mathbf{d}_{1}^{H}\right)$ is the cross-covariance matrix of $\mathbf{y}_{1}$ and $\mathbf{d}_{1}$. The matrices $\mathbf{R}_{\mathbf{d}_{1}}$ and $\mathbf{R}_{\mathbf{y}_{1}}$ are the auto-covariance matrices of $\mathbf{d}_{1}$ and $\mathbf{y}_{1}$, respectively. Let $\mathbf{I}_{H 1} \equiv \mathbf{H}_{1}^{H} \mathbf{I}_{1}, \mathbf{I}_{B 1} \equiv \mathbf{B}_{1} \mathbf{I}_{1}, \mathbf{R}_{\mathbf{I}_{H 1}}$ and $\mathbf{R}_{\mathbf{I}_{B 1}}$ are the auto-covariance matrices of $\mathbf{I}_{H 1}$ and $\mathbf{I}_{B 1}$, respectively. The cross-covariance matrix of $\mathbf{I}_{B 1}$ and $\mathbf{I}_{H 1}$ is $\mathbf{r}_{\mathbf{I}_{B 1} \mathbf{I}_{H 1}} \equiv E\left(\mathbf{I}_{B 1} \mathbf{I}_{H 1}^{H}\right)$. Then

$$
\mathbf{F}_{1} \equiv\left(\mathbf{R}_{\mathbf{I}_{H 1}}-\mathbf{r}_{\mathbf{I}_{B 1} \mathbf{I}_{H 1}}^{H} \mathbf{R}_{\mathbf{I}_{B 1}}^{-1} \mathbf{r}_{\mathbf{I}_{B 1} \mathbf{I}_{H 1}}\right) \text {. }
$$

Inserting (11) into (10) followed by some manipulation yields the output of the ML filter bank

$$
\mathbf{z}_{m l}=\mathbf{A}_{1} \mathbf{U}_{1}^{H} \mathbf{F}_{1}^{-1}\left[\mathbf{d}_{1}-\mathbf{r}_{\mathbf{y}_{1} \mathbf{d}_{1}}^{H} \mathbf{R}_{\mathbf{y}_{1}}^{-1} \mathbf{y}_{1}\right] \text {. }
$$

Comparing the multistage structure for the BLUE, MMSE and MOE filter banks $[2,3]$, we see that the ML detector possesses the same multistage structure, modulo a different output scaling matrix. The filters' soft outputs can be thus represented with a common form:

$$
\begin{aligned}
\mathbf{z}_{i} & \equiv \omega_{i} \mathbf{T y}=\mathbf{C}_{i}\left[\mathbf{d}_{1}-\mathbf{r}_{\mathbf{y}_{1} \mathbf{d}_{1}}^{H} \mathbf{R}_{\mathbf{y}_{1}}^{-1} \mathbf{y}_{1}\right] \\
i & \in\{\text { mmse, moe, blue, ml }\},
\end{aligned}
$$

with $\mathbf{C}_{m m s e} \equiv \mathbf{A}_{1} \mathbf{U}_{1}^{H} \mathbf{E}_{1}^{-1}, \mathbf{C}_{m o e} \equiv \mathbf{A}_{1} \mathbf{U}_{1}^{H}, \mathbf{C}_{\text {blue }} \equiv$ $\mathbf{A}_{1}^{-1} \mathbf{U}_{1}^{-1}$ and $\mathbf{C}_{m l} \equiv \mathbf{A}_{1} \mathbf{U}_{1}^{H} \mathbf{F}_{1}^{-1}$, where

$$
\mathbf{E}_{1}=\left(\mathbf{R}_{\mathbf{d}_{1}}-\mathbf{r}_{\mathbf{y}_{1} \mathbf{d}_{1}}^{H} \mathbf{R}_{\mathbf{y}_{1}}^{-1} \mathbf{r}_{\mathbf{y}_{1} \mathbf{d}_{1}}\right)=\mathbf{F}_{1}+\mathbf{U}_{1} \mathbf{A}_{1}^{2} \mathbf{U}_{1}^{H} .
$$




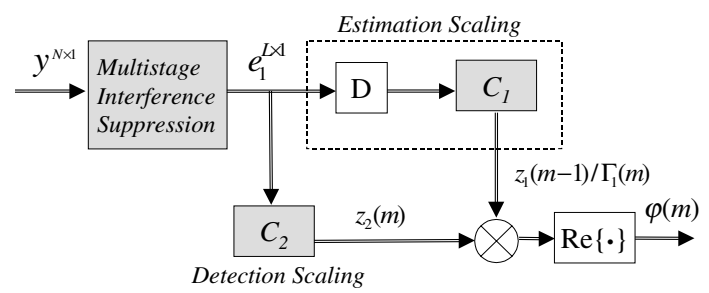

Fig. 2. The common framework for MRC and EGC detectors in multi-path fading channels. For MRC schemes, the estimation scaling is replaced by the true channel coefficient $\Gamma_{1} . C_{1}$ and $C_{2}$ could be the scaling matrices of MMSE/MOE/BLUE/ML-FB, in general.

\section{Common Framework for Blind Multistage DETECTION}

The methods of MRC and EGC combining can be considered in the context of coherent detection in multipath versus non-coherent detection in multipath. When the channel state is known, MRC is possible and the decision statistic for BPSK modulation is given by $\varphi(m)=\operatorname{Re}\left(\Gamma^{H}(m) \mathbf{z}(m)\right)$. In the absence of channel state information, we can employ differential DBPSK modulation and the resulting decision statistic is given by $\varphi(m)=\operatorname{Re}\left(\mathbf{z}^{H}(m-1) \mathbf{z}(m)\right)$, we denote this combining method EGC. In this scenario, the first soft estimate $\mathbf{z}(m-1)$ acts as the channel state estimate. A further flexibility afforded to us by the common framework of the various multistage filterbanks under consideration is that two different filterbanks can be employed for the implicit channel estimates and the filtered observation. Thus we propose heterogeneous combining methods based on the top performing MS FBs: $\varphi(m)=\operatorname{Re}\left(\mathbf{z}_{b l u e}^{H} \mathbf{z}_{m m s e}\right)$ and $\left.\varphi(m)=\operatorname{Re}\left(\mathbf{z}_{b l u e}^{H} \mathbf{z}_{m l}\right)\right)$, in contrast to the homogeneous schemes we previously proposed $[2,3]$.

From Fig. 1, it is clear that there is no need to implement individual filters for each of the BLUE, MMSE and ML estimators. Matrix $\mathbf{F}_{1}$ can be expressed as $\mathbf{F}_{1}=E\left[\left(\mathbf{H}_{1}^{H} \mathbf{I}_{1}-\omega_{1}^{H} \mathbf{B}_{1} \mathbf{I}_{1}\right)\left(\mathbf{H}_{1}^{H} \mathbf{I}-\omega_{1}^{H} \mathbf{B}_{1} \mathbf{I}_{1}\right)^{H}\right]$, where $\omega_{1} \equiv \mathbf{R}_{\mathbf{I}_{B 1}}^{-1} \mathbf{r}_{\mathbf{I}_{B 1} \mathbf{I}_{H 1}}=\mathbf{R}_{\mathbf{y}_{1}}^{-1} \mathbf{r}_{\mathbf{y}_{1} \mathbf{d}_{1}}$ is an MMSE filter bank as shown in Fig. 1. The physical meaning of $\mathbf{F}_{1}$ is essentially the autocorrelation matrix of the residual interference in the signal space, $\mathcal{H}_{1}$, filtered by $\omega_{1}$. The physical meaning of $\mathbf{E}_{1}$ is $\mathbf{E}_{1} \equiv E\left(\mathbf{e}_{1} \mathbf{e}_{1}^{H}\right)=\mathbf{F}_{1}+\mathbf{U}_{1} \mathbf{A}_{1}^{2} \mathbf{U}_{1}^{H}$, [cf. Fig. 1 and (15)], and $\mathbf{U}_{1} \mathbf{A}_{1}^{2} \mathbf{U}_{1}^{H}$ is the autocorrelation matrix of the projection of $\mathbf{S}_{1+} \mathbf{A}_{1}$ on the signal subspace, $\mathcal{H}_{1}$. Therefore, the multistage structure in Fig. 1 is essentially a multistage interference suppressor. A common framework for the implementation of the EGC receivers is developed in Fig. 2. Applying different scaling matrices for $\mathbf{C}_{1}$ and $\mathbf{C}_{2}$ yields different EGC receivers. We are in particular interested in $\mathbf{C}_{1}=\mathbf{C}_{\text {blue }}$ and $\mathbf{C}_{2}=\mathbf{C}_{m l}$ or $\mathbf{C}_{m m s e}$ for the aforementioned two heterogenous EGC receivers. The homogeneous EGC MMSE/MOE/BLUE receivers can also be implemented by having $\mathbf{C}_{1}=\mathbf{C}_{2}$ for each type of receiver.

We next analyze the BER performance for the common detection framework of Fig. 2. To that end, we first need to characterize the steady-state average SINR as well as the output SINR for the multistage MRC ML detector.

\section{AnAlysis of Output SINRs}

Since the multistage ML detector shares the same structure for the implementations of the MMSE/MOE/BLUE FBs, the subspace $\mathcal{T}_{L D}$ spanned by the D-stage
MMSE/MOE/BLUE/ML FBs is given by [2],

$\mathcal{T}_{L D} \operatorname{span} \mathbf{T}_{L D}^{H} \equiv\left[\mathbf{H}_{1}\left|\mathbf{H}_{2}\right| \ldots \mid \mathbf{H}_{D}\right] \equiv\left[\mathbf{H}_{1} \mid \mathbf{H}_{1 \perp}\right]$. (16)

The $\mathbf{E}_{1}$ term $[c f$. (15)] corresponding to the D-stage reduced-rank transformation $\mathbf{T}_{L D}$ is given by [2]

$\mathbf{S}_{D} \equiv \mathbf{H}_{1}^{H} \mathbf{R} \mathbf{H}_{1}-\mathbf{H}_{1}^{H} \mathbf{R} \mathbf{H}_{1 \perp}\left(\mathbf{H}_{1 \perp}^{H} \mathbf{R} \mathbf{H}_{1 \perp}\right)^{-1} \mathbf{H}_{1 \perp}^{H} \mathbf{R} \mathbf{H}_{1}$, (17) where the steady-state autocorrelation $\mathbf{R}$ is given by

$$
\mathbf{R}=\sum_{k=1}^{K} \mathbf{S}_{k+} \mathbf{A}_{k}^{2} \mathbf{S}_{k+}^{H}+\mathbf{S}_{k-} \mathbf{A}_{k}^{2} \mathbf{S}_{k-}^{H}+N_{0} \mathbf{I} .
$$

The $\mathbf{F}_{1}$ term $[c f$. (15)] corresponding to the D-stage implementation is given by $\mathbf{F}_{D} \equiv \mathbf{S}_{D}-\mathbf{U}_{1} \mathbf{A}_{1}^{2} \mathbf{U}_{1}^{H}$.

Ignoring self induced ISI, i.e. assume $\mathbf{S}_{1-}=\mathbf{0}$, the steady state average SINR and the output SINR of the MRC MS-ML-FB are given by,

Proposition 1: For the D-stage implementation of the multistage ML filter bank:

1. The average SINR is equal to

$$
S I N R_{m l}^{a v g}=\operatorname{tr}\left[\mathbf{A}_{1} \mathbf{U}_{1}^{H} \mathbf{F}_{D}^{-1} \mathbf{U}_{1} \mathbf{A}_{1}\right] .
$$

2. The output SINR is equal to

$$
S I N R_{m l}=\frac{t r^{2}\left[\mathbf{A}_{1} \mathbf{U}_{1}^{H} \mathbf{F}_{D}^{-1} \mathbf{U}_{1} \mathbf{A}_{1}\right]}{\operatorname{tr}\left[\mathbf{A}_{1} \mathbf{U}_{1}^{H} \mathbf{F}_{D}^{-1} \mathbf{U}_{1} \mathbf{A}_{1}+\left(\mathbf{A}_{1} \mathbf{U}_{1}^{H} \mathbf{F}_{D}^{-1} \mathbf{U}_{1} \mathbf{A}_{1}\right)^{2}\right]}
$$

We express the eigenvalue decomposition of the two kernel matrices as $K_{m m s e}=\mathbf{A}_{1} \mathbf{U}_{1}^{H} \mathbf{S}_{D}^{-1} \mathbf{U}_{1} \mathbf{A}_{1}=\mathbf{Q} \Lambda \mathbf{Q}^{H}$ and $K_{m l}=\mathbf{A}_{1} \mathbf{U}_{1}^{H} \mathbf{F}_{D}^{-1} \mathbf{U}_{1} \mathbf{A}_{1}=\mathbf{Q} \Lambda_{F} \mathbf{Q}^{H}$, where $\Lambda=$ $\operatorname{diag}\left(\lambda_{1}, \lambda_{2} \ldots \lambda_{L}\right)$ and $\Lambda_{F}=\operatorname{diag}\left(\lambda_{f_{1}}, \lambda_{f_{2}} \ldots \lambda_{f_{L}}\right)$. It can be shown that the two eigenvalues sets are related by:

$$
\Lambda_{F}=\Lambda(\mathbf{I}-\Lambda)^{-1}=\Lambda+\Lambda^{2}+\Lambda^{2} \cdots .
$$

The performance deviation between the output SINRs of the D-stage MRC MMSE and ML filter banks is given by:

Proposition 2: For the D-stage MMSE/ML FBs,

$$
\begin{gathered}
S I N R_{m m s e}-S I N R_{m l}= \\
\frac{\sum_{i=1}^{L-1} \sum_{j=i+1}^{L} \lambda_{i} \lambda_{j}\left(\prod_{l=1, l \neq i, j}^{L}\left(\lambda_{l}+1\right)\right)\left(\lambda_{i}-\lambda_{j}\right)^{2}}{\prod_{j}^{L}\left(\lambda_{j}+1\right) \sum_{i}^{L} \lambda_{i}\left(\lambda_{i}+1\right)} \geq 0 .
\end{gathered}
$$

Since $L \geq S I N R_{\text {mmse }}$, [3], thus $L \geq S I N R_{m m s e} \geq$

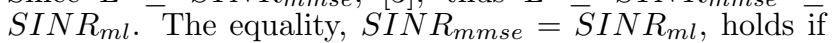
and only if $\Lambda_{F}=\kappa \mathbf{I}, \kappa>0$, i.e. $\mathbf{A}_{1} \mathbf{U}_{1}^{H} \mathbf{F}_{D}^{-1} \mathbf{U}_{1} \mathbf{A}_{1}=\kappa \mathbf{I}$.

\section{Generic BER Formula}

To obtained a closed-form BER formula for the common detection framework of Fig. 2, we extend the approach presented in [7] where the BER is expressed in terms of the residue of a moment generating function. Define matrix $J=\frac{1}{2}\left[\begin{array}{ll}\mathbf{0} & \mathbf{I} \\ \mathbf{I} & \mathbf{0}\end{array}\right]$ and $\mathbf{x}=\left[\begin{array}{ll}\mathbf{g}^{H} & \mathbf{z}(m)^{H}\end{array}\right]^{H}$. The vector $\mathbf{g}$ is equal to $\Gamma_{1}$ for MRC filter banks or equal to $\mathbf{z}(m-1)$ for EGC filter banks. The decision statistics can thus be rewritten as $\varphi(m)=\operatorname{Re}\left[\mathbf{g}^{H} \mathbf{z}(m)\right]=\mathbf{x}^{H} J \mathbf{x},[c f$. Fig. 2]. Notice that $\mathbf{z}(m-1)$ and $\mathbf{z}(m)$ could be the outputs of two different types of filters. The moment generating function is defined as $M(s) \equiv E\left[e^{s \varphi}\right]=[\operatorname{det}(\mathbf{I}+s J \Phi)]^{-1}$, where $\Phi=E\left(\mathbf{x x}^{H}\right)$. The BER is given by

$$
\operatorname{Pr}\left(\mathbf{x}^{H} \mathbf{J} \mathbf{x}<0\right)=-\sum_{R H P} \operatorname{Res}\left\{\frac{M(s)}{s}\right\} .
$$

Now, from (23), it suffices to derive the moment generating function $M(s)$ for each filter bank. Due to the 
fact that filter banks including MMSE/MOE/BLUE/ML satisfy a symmetry property of the form: $\omega_{i}^{H} \mathbf{T} \mathbf{S}_{1+} \mathbf{A}_{1}$ is Hermitian, a signal correlation matrix $\mathbf{E}_{S_{i}}$ and an output correlation matrix $\mathbf{E}_{O_{i}}$ can be defined for these filter banks $\omega_{i}$ :

$$
\begin{aligned}
\mathbf{E}_{S_{i}} & \equiv \omega_{i}^{H} \mathbf{T}_{L D} \mathbf{R}_{S} \mathbf{T}_{L D}^{H} \omega_{i}=\left(\omega_{i}^{H} \mathbf{T}_{L D} \mathbf{S}_{1+} \mathbf{A}_{1}\right)^{2} \\
\mathbf{E}_{O_{i}} & \equiv \omega_{i}^{H} \mathbf{T}_{L D} \mathbf{R} \mathbf{T}_{L D}^{H} \omega_{i}
\end{aligned}
$$

where $\mathbf{R}_{S} \equiv \mathbf{S}_{1+} \mathbf{A}_{1}^{2} \mathbf{S}_{1+}^{H}$. Furthermore, we define $\mathbf{E}_{S} \equiv$ $\mathbf{E}_{S_{1}}^{\frac{1}{2}} \mathbf{E}_{S_{2}}^{\frac{1}{2}}$ and $\mathbf{E}_{O} \equiv \mathbf{E}_{O_{1}}^{\frac{1}{2}} \mathbf{E}_{O_{2}}^{\frac{1}{2}}$. A generic formula for $M(s)^{-1}$ is proposed following the form

$$
\begin{aligned}
\operatorname{det}[\mathbf{I}+s \mathbf{J} \Phi]= & \operatorname{det}\left[\mathbf{I}-\frac{s}{2}\left(\mathbf{E}_{O_{1}}^{\frac{1}{2}} \mathbf{E}_{O_{2}}^{\frac{1}{2}}-\rho \mathbf{E}_{S_{1}}^{\frac{1}{2}} \mathbf{E}_{S_{2}}^{\frac{1}{2}}\right)\right] \times \\
& \operatorname{det}\left[\mathbf{I}+\frac{s}{2}\left(\mathbf{E}_{O_{1}}^{\frac{1}{2}} \mathbf{E}_{O_{2}}^{\frac{1}{2}}+\rho \mathbf{E}_{S_{1}}^{\frac{1}{2}} \mathbf{E}_{S_{2}}^{\frac{1}{2}}\right)\right] .
\end{aligned}
$$

For MRC schemes, the inverse of $M(s)$ is obtained by setting $\mathbf{E}_{S_{1}}=\mathbf{E}_{O_{1}}=\mathbf{I}$ and $\rho=1$. We can show that this formula holds for the following receiver structures: MRC MMSE/BLUE/ML FBs, homogeneous EGC MMSE/MOE/BLUE/ML FBs or heterogeneous EGC BLUE-MMSE and BLUE-MOE FBs. Absent from this list is the MRC MOE-FB; where the result holds for the flat Rayleigh fading case, but cannot be shown explicitly for the multipath channel case. We note that an empirical comparison of the appropriately computed formula and simulations show close agreement; however that the equation in fact holds for the MRC MOE scenario is just conjecture at this point.

The BERs of the reduced-rank MRC and EGC BLUEMMSE and BLUE-ML schemes are listed in Table I. As Table I shows, the BERs of either the MRC MMSE or EGC BLUE-MMSE receivers can be expressed in terms of of $\Lambda$ whose trace is the output SINR of the reduced-rank MRC MMSE-FB in the sense of (7). On the other hand, BERs of the MRC-ML or EGC BLUE-ML receivers can be expressed in terms of $\Lambda_{F}$ whose trace is the average SINR of the reduced-rank MRC ML-FB in the sense of (5). The major difference between the BERs of BPSK and DBPSK is that the autocorrelation value $\rho$ of the fading process appears explicitly in the BER formulae for DBPSK.

Numerical simulations are conducted to verify the formula (26). A short-code (Gold Code) DS-CDMA system with spreading gain $N=31$ is considered. Each user experiences a 3-path (i.e. $L=3$ ) Rayleigh fading channel with normalized Doppler shift $f_{d} T_{s}=5 \times 10^{-3}$. All users are assumed to have the same powers. The path delay $\tau_{k l}$ for each user is uniformly distributed over $\left[0,6 T_{c}\right)$ to constrain the strength of ISI which is ignored in the above analysis for convenience. The BERs of the MRC MMSE/BLUE/MLFBs for BPSK modulated DS-CDMA systems and that of the heterogeneous EGC BLUE-MMSE and BLUE-ML filter banks as well as the homogeneous EGC BLUE-BLUE, [3], for non-coherent detection of DBPSK are presented in Fig. 3. For the analysis of MRC schemes, only the desired user's ISI is ignored, but for EGC, all users's ISIs are ignored. Thus the analytic MRC results are more accurate than that of the EGC schemes.

\section{Limiting Performance}

We observe in Fig. 3 that both EGC and MRC schemes suffer from BER floors at high SNR. Let $D_{\text {free }}=(D-1) L$ indicate the degrees of freedom afforded by the reducedrank MS FB of interest; we further defined $D_{\text {int }}=$ $\operatorname{rank}\left(E\left[(M A I+I S I)(M A I+I S I)^{H}\right]\right)$ to be the dimension of the interference without noise. We have shown in

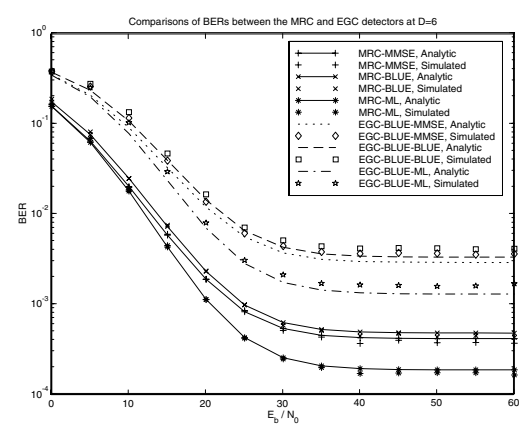

Fig. 3. Performance comparisons of BERs for the EGC BLUE-ML$\mathrm{FB}$ and BLUE-MMSE-FB vs. the MRC ML-FB and MMSE-FB. $K=10, N=31, L=3$. The number of applying stages is $D=6$.

[4] that if $D_{\text {free }} \geq D_{\text {int }}$, then the MRC type receivers will not experience a BER floor. A natural question to ask, which we answer herein, is what is the needed relationship for the EGC schemes to avoid BER floors. We shall show that the EGC schemes will always experience BER floors at high SNR even if $D_{\text {free }} \geq D_{\text {int }}$; this is the price of imperfect channel state information. As an exemplar, we evaluate the limiting performance of the EGC-MMSE detector structure. Similar phenomena apply to other EGC schemes, since they all share the same multistage structure.

Ignoring ISI, the second term on the right hand side of (18), the approximate auto-correlation matrix is

$$
\tilde{\mathbf{R}}=\mathbf{S}_{1+} \mathbf{A}_{1}^{2} \mathbf{S}_{1+}^{H}+\sum_{k=2}^{K} \mathbf{S}_{k+} \mathbf{A}_{k}^{2} \mathbf{S}_{k+}^{H}+N_{0} \mathbf{I} \equiv \mathbf{R}_{S}+\mathbf{R}_{I}
$$

where $\mathbf{R}_{S}=\mathbf{S}_{1+} \mathbf{A}_{1}^{2} \mathbf{S}_{1+}^{H}$ and $\mathbf{R}_{I}=\sum_{k=2}^{K} \mathbf{S}_{k+} \mathbf{A}_{k}^{2} \mathbf{S}_{k+}^{H}+$ $N_{0} \mathbf{I}$. Define the interference covariance matrix $\mathbf{N}_{I}=$ $\omega^{H} \mathbf{T R}_{I} \mathbf{T}^{H} \omega$. Thus, $\mathbf{E}_{O}=\omega^{H} \mathbf{T R T}^{H} \omega \simeq \omega^{H} \mathbf{T}\left(\mathbf{R}_{I}+\right.$ $\left.\mathbf{R}_{S}\right) \mathbf{T}^{H} \omega=\mathbf{E}_{S}+\mathbf{N}_{I}$. Eq. (26) can be rewritten as

$$
\begin{aligned}
\operatorname{det}[\mathbf{I}+s \mathbf{J} \Phi]= & \operatorname{det}\left[\mathbf{I}-\frac{s}{2}\left((1-\rho) \mathbf{E}_{S}+\mathbf{N}_{I}\right)\right] \times \\
& \operatorname{det}\left[\mathbf{I}+\frac{s}{2}\left((1+\rho) \mathbf{E}_{S}+\mathbf{N}_{I}\right)\right] .
\end{aligned}
$$

Substituting the expression for $\omega$ of the MS MMSE-FB, we have $\mathbf{E}_{S}=\mathbf{Q} \Lambda^{2} \mathbf{Q}^{H}$ and $\mathbf{E}_{O}=\mathbf{E}_{S}^{\frac{1}{2}}$. Thus, $\mathbf{N}_{I}=\mathbf{Q}(\Lambda-$ $\left.\Lambda^{2}\right) \mathbf{Q}^{H}$. Substituting these terms back into (28) yields

$$
\operatorname{det}[\mathbf{I}+s \mathbf{J} \Phi]=\operatorname{det}\left[\mathbf{I}-\frac{s}{2}\left(\Lambda-\rho \Lambda^{2}\right)\right] \operatorname{det}\left[\mathbf{I}+\frac{s}{2}\left(\Lambda+\rho \Lambda^{2}\right)\right] .
$$

Since $\Lambda<\mathbf{I}$ and $\rho<1$, the first determinant on the right hand side determines the RHP poles. Due to the fact that multipath do not make receivers any physically different, and as will be shown that BERs for any type of EGC combining schemes are equal in flat fading channels ${ }^{1}$. To highlight key properties, we discuss the case of flat fading channels. Thus, $0 \leq E_{S}=\lambda^{2} \leq 1$, and $0 \leq N_{I}=\lambda-\lambda^{2} \leq \frac{1}{4}$. The upper bound of $N_{I}$ can be easily obtained by taking its derivative with respect to $\lambda$ and setting it to zero. By $(21), 0 \leq \frac{E_{S}}{N_{I}}=\frac{\lambda}{1-\lambda}=\lambda_{f}=\lambda+\lambda^{2}+\cdots \leq \infty$. At high $\mathrm{SNR}, \frac{N_{I}}{E_{S}}=\frac{1}{\lambda_{f}} \ll 1$. The BER of EGC receiver can be manipulated into the form of

$$
B E R_{E G C}=\frac{(1-\rho \lambda)}{2}=\frac{(1-\rho) \lambda^{2}+\lambda-\lambda^{2}}{2\left(\lambda^{2}+\lambda-\lambda^{2}\right)}
$$

${ }^{1}$ This coincides with the fact that output SINRs of all MRC MMSE/MOE/BLUE/ML FBs are the same in flat fading channels $[4]$. 


\begin{tabular}{|c|c|}
\hline Combining & BERs for MRC MMSE and ML Receivers \\
\hline MMSE & $-\sum_{R H P} \operatorname{Res}\left(s \cdot \operatorname{det}\left[\mathbf{I}-\frac{s}{2}\left(\Lambda^{\frac{1}{2}}-\Lambda\right)\right] \operatorname{det}\left[\mathbf{I}+\frac{s}{2}\left(\Lambda^{\frac{1}{2}}+\Lambda\right)\right]\right)^{-1} ; \mathbf{E}_{S}=\left(\mathbf{A}_{1} \mathbf{U}_{1}^{H} \mathbf{S}_{D}^{-1} \mathbf{U}_{1} \mathbf{A}_{1}\right)^{2}$ \\
\hline ML & $-\sum_{R H P} \operatorname{Res}\left(s \cdot \operatorname{det}\left[\mathbf{I}-\frac{s}{2}\left(\left(\Lambda_{F}+\Lambda_{F}^{2}\right)^{\frac{1}{2}}-\Lambda_{F}\right)\right] \operatorname{det}\left[\mathbf{I}+\frac{s}{2}\left(\left(\Lambda_{F}+\Lambda_{F}^{2}\right)^{\frac{1}{2}}+\Lambda_{F}\right)\right]\right)^{-1} ; \mathbf{E}_{S}=\left(\mathbf{A}_{1} \mathbf{U}_{1}^{H} \mathbf{F}_{D}^{-1} \mathbf{U}_{1} \mathbf{A}_{1}\right)^{2}$ \\
\hline Combining & BERs for EGC BLUE-MMSE and BLUE-ML Receivers \\
\hline BLUE-MMSE & $-\sum_{R H P} \operatorname{Res}\left(s \cdot \operatorname{det}\left[\mathbf{I}-\frac{s}{2}(\mathbf{I}-\rho \Lambda)\right] \operatorname{det}\left[\mathbf{I}+\frac{s}{2}(\mathbf{I}+\rho \Lambda)\right]\right)^{-1} ; \mathbf{E}_{S}=\left(\mathbf{A}_{1} \mathbf{U}_{1}^{H} \mathbf{S}_{D}^{-1} \mathbf{U}_{1} \mathbf{A}_{1}\right)$ \\
\hline BLUE-ML & $-\sum_{R H P} \operatorname{Res}\left(s \cdot \operatorname{det}\left[\mathbf{I}-\frac{s}{2}\left(\mathbf{I}+\Lambda_{F}-\rho \Lambda_{F}\right)\right] \operatorname{det}\left[\mathbf{I}+\frac{s}{2}\left(\mathbf{I}+\Lambda_{F}+\rho \Lambda_{F}\right)\right]\right)^{-1} ; \mathbf{E}_{S}=\left(\mathbf{A}_{1} \mathbf{U}_{1}^{H} \mathbf{F}_{D}^{-1} \mathbf{U}_{1} \mathbf{A}_{1}\right)$ \\
\hline
\end{tabular}

TABLE I

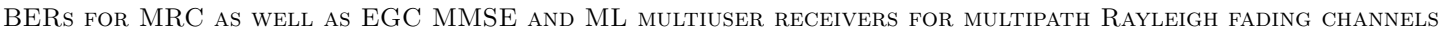

$$
\begin{aligned}
& =\frac{(1-\rho)}{2\left(1+N_{I} / E_{S}\right)}+\frac{N_{I} / E_{S}}{2\left(1+N_{I} / E_{S}\right)} \\
& \simeq \frac{(1-\rho)}{2}+\frac{N_{I} / E_{S}}{2} \text { as } \frac{N_{I}}{E_{S}} \ll 1 .
\end{aligned}
$$

This shows that the BER is controlled by two factors, the first term is due to the channel mismatch between two adjacent symbol intervals and the second term is related to $E_{S} / N_{I}$. So, even if $D_{\text {free }} \geq D_{\text {int }}$ at high SNR such that $\lambda \sim 1$ and $\frac{E_{S}}{N_{I}}=\lambda_{f} \sim \infty$ [4], a differential EGC scheme still suffers from a BER floor due to the auto-correlation value of the fading process. We also note that (30) has the same form of the BER formula to DBPSK of non-spread systems. The difference is that $E_{S} / N_{I}$ is replaced by $E_{b} / N_{0}$ for DBPSK demodulation, where $E_{b}$ and $N_{0}$ are the signal and the noise variances, respectively. In our case, $E_{S}$ and $N_{I}$ are the filtered signal and filtered interference variances.

The BER for all MRC schemes are the same in flat fading given by

$$
B E R_{M R C}=\frac{1-\lambda^{\frac{1}{2}}}{2} .
$$

As a result, the BER for MRC schemes is a function of the eigenvalue $\lambda$ only. When $D_{\text {free }} \geq D_{\text {int }}, \lambda \rightarrow 1$ as $E_{b} / N_{0}$ keeps increasing. So $B E R_{M R C}$ decreases without hitting a floor. Fig. 4 illustrates this point by presenting the BERs of the EGC BLUE-MMSE and BLUE-ML schemes versus the BERs of the MRC-MMSE and MRC-ML schemes. In this simulation, $K=10, N=31, D_{\text {int }}=18, f_{d} T_{s}=$ $5 \times 10^{-3}$ and $\rho=J_{0}\left(2 \pi f_{d} T_{s}\right)=0.99975$. For full-rank schemes, $D_{\text {free }}-D_{\text {int }}=12$. The BERs of EGC schemes hit a floor level of $0.5 \times(1-\rho)=1.25 \times 10^{-4}$. On the other hand, for the reduced-rank schemes, $D_{\text {free }}-D_{\text {int }}=$ -7 . $\lambda$ saturates at a value $\lambda_{\text {sat }} \simeq 0.9998$ due to residual interference. This SINR saturation yields a BER floor at $0.5 \times\left(1-\lambda_{\text {sat }}^{\frac{1}{2}}\right) \simeq 5 \times 10^{-5}$ for the MRC schemes and a BER floor at $0.5 \times\left(1-\rho \lambda_{\text {sat }}\right) \simeq 2.25 \times 10^{-4}$ for the EGC schemes. Similar arguments also explain the limiting performance phenomena for multipath fading channels as demonstrated in Fig. 3, where $D_{\text {free }}=15$ and $D_{\text {int }}=31$.

\section{Conclusions}

In this paper, we have developed a common framework for the multistage implementation of MRC and EGC MMSE/MOE/BLUE/ML filterbanks operating in multipath channels. We have generalized our prior work to include ML based filterbanks as well as generalizing receiver structures for EGC methods to allow for heterogeneous EGC which can yield performance improvements over their homogeneous counterparts. A key feauture of our general

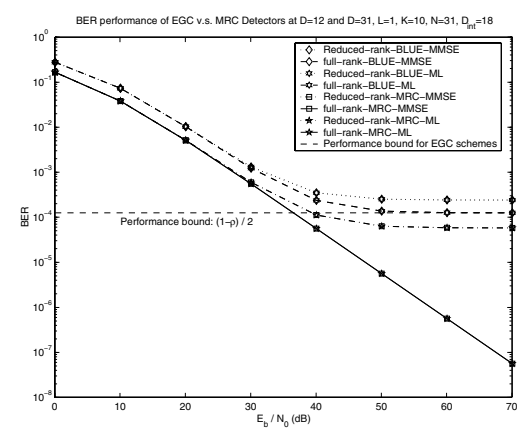

Fig. 4. Performance comparisons of BERs for the EGC BLUE-MLFB and BLUE-MMSE-FB vs. the MRC ML-FB and MMSE-FB over a flat Rayleigh fading channel with $f_{d} T_{s}=5 \times 10^{-3}$. Reduced-rank schemes with $D=11$ and the full-rank ones are shown in this figure.

framework is that it facilitates a sytematic determination of the BER associated with each of these receiver structures in fading multipath channels. Furthermore, the BER evaluation clearly shows why both MRC and EGC schemes experience BER floors at high SNRs and also provides a condition for avoiding BER floors in MRC methods.

\section{REFERENCES}

[1] J. S. Goldstein, I. S. Reed, and L. L. Scharf, "A multistage representation of the wiener filter based on orthogonal projections," IEEE Trans. on Information Theory, vol. 44, no. 7, pp. 2943IEEE Trans. on
2959, Nov. 1998.

[2] S.-H. Wu, U. Mitra, and C.-C. Kuo, "Multi-stage MMSE/MOE receivers for frequency-selective fading channels in DS-CDMA systems," in Proc. IEEE Globecom. Taipei, Taiwan, Nov 2002.

[3] S.-H. Wu, U. Mitra, and C.-C. Kuo, "Perfprmance analysis of multistage BLUE/MMSE receivers for DS-CDMA in frequencyselective fading channels," in Proc. IEEE ICC. Alaska, May 2003, to be presented.

[4] S.-H. Wu, U. Mitra, and C.-C. Kuo, "Performance analysis of a class of multistage DS-CDMA receivers for multipath channels," in Proc. IEEE Information Theory Workshop (ITW). Paris, French, April 2003, to be presented.

[5] M. L. Honig and J. S. Goldstein, "Adaptive reduced-rank interference suppression based on the multistage wiener filter," IEEE Trans. on Communications, vol. 50, no. 6, pp. 986-994, June 2002.

[6] M. L. Honig and Weimin Xiao, "Performance of reduced-rank linear interference suppression," IEEE Trans. on Information Theory, vol. 47, no. 5, pp. 1928-1946, July 2001.

[7] S. L. Miller, M. L. Honig, and L. B. Milstein, "Performance analysis of MMSE receivers for DS-CDMA in frequency-selective fading channels," IEEE Trans. on Communications, vol. 48, no. 11, pp. 1919-1929, Nov. 2000

[8] U. Madhow and M. L. Honig, "MMSE interference suppression for direct-sequence spread-spectrum CDMA," IEEE Trans. on Communications, vol. 42, no. 12, pp. 3178-3188, Dec. 1994.

[9] S. Haykin, Adaptive Filter Theory, Prentice Hall, 3rd edition, 1996. 